\title{
The Emotive Component in English-Russian Translation of Specialized Texts
}

\author{
Natalia Sigareva \\ The Herzen State Pedagogical University of Russia, Saint Petersburg, Russia
}

\begin{abstract}
In this paper, the author will focus on the emphatic component of specialized texts and discuss some issues of intercultural communication effectiveness in diverse socio-cultural context. Many emotional theories have already placed the major emphasis on investigation of cultural dimensions of emotions. It has been stated that emotions are greatly determined by the systems of cultural values. The way the emotions are displayed (or hidden) in different languages is crucial for verbalization of different emotional experiences. Thus, the translation techniques employed to bridge the communication between the selected cultures are of paramount importance in terms of cultural norms and regulations characteristic of different lingual communities. The author will argue that translation of technical texts from English into Russian presents a challenge from the point of view of consensual ideology which differs in respectful cultures in the discussed domain.
\end{abstract}

Keywords: socio-cultural context, emotive component, translation of specialized texts, translator competence

\section{Introduction}

It has been identified on the national level as the Russian economy becomes more closely integrated with the other countries of the world and in particular with the countries of the European Union, the need for trained professionals in translation of technical texts has been repeatedly noted by the relevant services in Russia and international organizations. The research was based on the experience acquired through the project training at Herzen University. The main result was to develop a consistent, coherent, and coordinated methodology of training that would determine the translation market value, providing a solid theoretical foundation to equip the students with conceptual tools, and giving them an ample opportunity to place more importance to the practical side of training. Special stress was laid on the socio-cultural approach of training which means acqusition of tricks from experts and interaction between participants of the group. Interdisciplinary approach was implemented in order to optimize the different types of learning. The learning process has to be active, linked to the situation and imbedded in a social context. University trainers create authentic professional situations involving students into real translation projects, which have the advantage to stimulate the motivation of the students to enlarge their theoretical background. The challenges of translation of scientific texts from English into Russian reveal not only the importance of documentary reseach and

Natalia Sigareva, assistant professor, Department of Translation and Interpreting, The Herzen State Pedagogical University of Russia. 
creation of terminology equivalence in a certain technical field but the importance of the culture behind the language for accurate translation. The students should be trained to link concepts and languages aiming to relay a message across cultural and linguistic boundaries.

\section{Discussion}

When analyzing technical texts, referential function is typically brought into focus. The reason for that is the assumption that in specialist texts, the language is mainly used to identify an object, a process, or a state of the reality. These texts are usually characterized by the frequent use of the passive voice, subject pronoun "it", abstract nouns, and nominalization. Besides, the personal perception of the topic by the text producer is not vivid. Impersonality is on the surface of objectivity, accentuating the seriousness of the point putting forward the meaning of the message, but not the form. Sending the message, the writer is concerned with the accuracy and obviousness of the described phenomena.

These characteristics seem to be universal. Nevertheless, the conventions of appropriateness in English and Russian do not coincide. The degree to which the use of language focuses on the perception of the producer-an emotive function - is different in this pair. Word play, metaphors, emotional words, and marked syntactical forms are thought to change the register and the level of formality in Russian texts. Classifying it as subjective (i.e., not scientific), Russian conventions restrict the use of emotive language in technical texts, whereas for English writers it is quite natural to use, say, metaphors in scientific discourse.

The tension between source and target texts is usually expressed in terms of equivalence, but when we consider how specialized text shifts between referential and emotive functions, the primary difficulty in translation derives from clashes in perception of the emotive aspect of objectivity.

Challenge posed by different approaches in the representation of the objective reality may involve translator adapting or altering the mode or the register as he/she shifts from English to Russian. The translators need to ensure that the text they produce is appropriate to target readership. The result of misinterpreting the emotive implication in the source text is one problem, another one, less vivid, but more important is the result of giving not accessible tone to the target text. There are many occasions when translators have to translate a relatively emotional pattern in a specialized text and consider the relevance of the emotive component in translation. This is the area where experience of a specialized text translator is vital.

The translator competence resulting in translation competence is achieved by consistent training in classifying texts in terms of similarities and differences and by recognizing these distinctions. The key point in making the target text accessible is that the translator should be able to: (1) compare the differences; (2) find what will make the text incoherent or awkward if cultural values are not being met; (3) reflect the cultural context which is to be processed; and (4) avoid shifting the focus to the form of the message away from meaning itself when preserving the emotive component in restricted domain (Russian technical texts).

\section{Results}

The study concludes that the translation process is a multiple-dimensional, purposeful with pragmatic principles and cross-cultural communication to be taken into consideration. 
Language is unquestionably rooted in the reality of culture and cannot be explained without reference to the broader contexts of verbal utterance. The translation should be taken not as an equivalence-based language replacement, but as a communication process mediation.

Language is a reflection of the system of cultural values and conventions acceptable, but even the profound knowledge of the two languages cannot guarantee the effective communication between two cultures. There is great demand in profound psychological and emotional involvement. A translator must take into account not only the intellectual, but also the emotional response that the target text may evoke in its reader. The failure to observe the conventions of emotive component usage in the target language may hinder the successful communication. Translators are expected to manipulate the source text in various ways in order to make it compatible with the requirements set by the target reader.

One of the challenges the English-Russian translator faces translating stylistically marked elements in English technical texts is the search for acceptable Russian equivalents that would not only express the same meaning, but also perform the same emotive function in the text, meet the lingua-ethnic competence of the readership, and fit the stylistic norm of the target text. Otherwise, the translators will come out with inappropriate, awkward, and ambiguous translations. The degree to which they can effectively transfer the message is the question of translation competence and the quality of the final product.

The linguistic base for sense in general is just a part of a whole. Readers/addressees apply relevant knowledge to complete it. Communication strategies are closely bound up with cultural beliefs, values, and expectations. Translators create a new balance between explicit and implicit elements. The translator shows an image identical to the one evoked in the original by creation of equivalence being faithful to the writer when building the new universe of knowledge in the target text.

The premise that language is metaphorically biased raises the problem of metaphor awareness approach in technical translation. The weight of metaphor in source and target texts is different, and the translators have to take into consideration the metaphoric perspective. Metaphor should be viewed as both the conceptual and linguistic device to deliver the meaning and the rhetorical move to achieve the effectiveness of communication.

Besides, emotionally colored adjectives widely used in English specialized texts are usually substituted by neutral equivalents in Russian texts. Raising awareness regarding the conventional usage of the target text is the major point in translator training.

Translation presupposes the involvement of emotions and power relations. To make the communication successful means to resort to cognitive and attitudinal resources. The most challenging for inter-delivery and for translation is the science translation of technical texts which shows its specific regulations in terms of the process of translation of cultural component.

Experience has shown that the corresponding and accurate meaning of a technical term or lexical unit used in the source science text can be easily provided by dictionaries but hardly ever used directly without pragma-cultural perspective as the main focus. The latter, finally, as a control variable presupposes the analytic diversity of meanings, involving textual tension and cultural factors that become concrete causations of change and transformation of the content. Genre as a control variable in cross cultural communication affects the translation language and hence the target culture: composition, conventions, and norms. 
In the hope of finding out the relevance between the linguistic formalization and explication in translation and bearing in mind the laws of human beings' cognitive processing, the translators have to serve readability, fidelity, terminology, and syntax as reasons for explication. Target texts dimensions in this respect are termed as acceptability, adequacy, and accuracy.

A translator is supposed to find answers to questions: Why does the writer write this? And: What cultural adaptation strategies are acceptable in this very situation for this particular recipient?

A broader description of the translation competence more emphasizing its communicative aspect involves transfer competence: the awareness of socially and culturally relevant context in processing of different linguistic codes and modalities. The transfer competence means the ability to understand the nuances and the ability to sensitively treat the cultural aspects, with the precision in rendering the message, precision in terminology expression as an absolute necessity.

Thus, to bridge the absence of equivalent typologies across cultures, a translator has to employ translation competence, that is to justify own decisions, exercise correct terminology-completeness of rendition, and clarity of expression; be aware of language change (language competence); develop the ability to identify functions and meaning, implicit content, cultural elements, to reconstruct the gist according to genre conventions (intercultural competence).

The acquisition of the main methodological, occupational, and psycho-physiological competences will enable a translator to map source text relations onto target text relations and exploit a series of standards that a text has to meet to be considered as such and recognized as textual communication.

To translate the emotive component found in English scientific texts into Russian is to encounter challenges to transfer both the proper intention and the meaning which call for full and accurate comprehension and appropriate and correct rendering. One of the most distinct features of Russian technical text is relatively infrequent use of emphatic structures because of this a translator may take this seemingly irrelevant for Russian scientific discourse element as subject to drop it out , sometimes resulting in the loss of meaning. The question "how to convert the prominent emotive elements in English texts into secondary elements in Russian translation in order to highlight the idea and achieve the purpose" is crucial.

Any particular instance of emphasis should be treated as obligatory or optional - two main categories distinguished in the translation model. Variation in the use of translation strategy is addressed in terms of individual, convention, and readership. The theory of risk avoidance by Pym (1992) puts forward the idea of developing translator's awareness of his/her place in a particular translating environment. It is very important to separate out what is universal from the conventions of translating in particular cultures.

The question of cultural transfer in scientific/technical translation from English into Russian and back necessitates the mechanisms of both conceptual and linguistic adjustment on the part of receiving culture. When we weigh translation decisions over effectiveness being equipped with various tools to overcome specific translation problems, we also have to trust our knowledge of cultural universals, raise comparative cultural and ethnographic awareness, and possess the ability to control the empathy. By comparing the similarities and differences of the two languages, the translator develops the skills that help to achieve the satisfactory result.

The production of an unambiguous translation can be achieved by means of understanding of peculiarities of 
discourse types and text genres, their structure, stylistic features, and discourse markers. Translation as rendering of the sentences at discourse level should take into consideration different textual dimensions: content, intention, and cultural variability of the factors influencing the meaning. Translating the culture that gives the meaning is required to effect successful translation.

To sum up, we have to admit that mediation, linguistic, cultural competences as well as the factual and research competences lay the basis for the strategic competence that helps achieve the best possible quality of translation.

The strategic competence reflects the environmental relativism in translation. Ecological principle in translation calls for translation process being as natural as possible, creating the comfortable environment for both cultures where the words and sentences are not disjoined, they create the world harmony of message delivery.

\section{Conclusions}

This short investigation has sought to show two basic points. First, not only the connotation of the emotion challenges the translation, but also the emotive function itself. The problem results from marked formality Russian technical texts are characterized by in comparison with the English tradition to resort to the use of emotive function by using different figures of speech. Second, as Russian, restrictions to use emotional language in scientific discourse are pretty rigid, a translator has to identify each type of referential and emotive function treating the text as the single unit and to analyze the impact of any change, negligence, or preservation of the cultural emotive accents.

The competent translator is always capable, on one hand, to put the extent to what the texts can be regarded as equivalent across cultures at stake, and, on the other hand, to raise awareness to what extent the translation decisions reflect the absence of the emotive component in target text.

In examining the intricacies of the translation process, we looked upon it as an act of communication in which the translator is at one and the same time a receiver and a producer of a text. The professional world requires that communication proceed efficiently and expects from a professionally trained translators a combination of effectiveness and efficiency in the form of translator competence which is regarded as textual competence and presents the acquisition of linguistic skills, world knowledge, and experience.

We have considered the competences related to the principles and strategies that have to be applied in order to work through the translation process and to be able to arrive at the appropriate decision. The developed skills should serve the way to grasp principles related to translation practice.

The translation competence should be treated in terms of ecological process, meaning that translation process goes as natural as possible, thus creating the universe of knowledge that presents a harmonious, effectively working system in the respectful cultures.

\section{References}

Alves, F., \& Gonçalves, J. L. (2006). A relevance theory approach to inferential processes in translation. In F. Alves (Ed.),

Triangulating translation. Amsterdam and Philadelphia: John Benjamins.

Bell, R. (1991). Translation and translating: Theory and practice. New York: Longman. 
European Committee for Standardisation. (2006). Nederlandse norm NEN-EN 15038 (en): Translation services: Service requirements. Brussels: Nederlands Normalisatie-instituut.

Hatim, M., \& Mason, I. (1900). Discourse and the translator. New York: Longman.

Lederer, M. (2003). The interpretive model. Manchester: St. Jerom.

Nord, C. (2005). Text analysis in translation: Theory, methodology, and didactic application of a model for translation-oriented text analysis. Amsterdam, New York: Rodopi.

Pattison, A. (2009). The Herzen "Human Technology” editing project. Modern concepts of university education. St. Petersburg: Herzen University.

Pym, A. (1992). Translation and text transfer: An essay on the principles of intercultural communication. Frankfurt, Main: Peter Lang. 